\title{
A Compound Blind Digital Watermarking Technique for Image Authentication and Integrity using DCT and VC
}

\author{
Inder Pratap \\ Research Scholar \\ Sri Balaji Technical Campus \\ Jaipur
}

\author{
Saroj Hiranwal \\ Assistant Professor \\ Sri Balaji Technical Campus \\ Jaipur
}

\author{
Dr. N.S.Yadav \\ Associate Professor \\ Sri Balaji Technical Campus \\ Jaipur
}

\begin{abstract}
In this paper we propose a multiple digital watermarking scheme using DCT and VC to achieve both image authentication and integrity. We used two watermarks for embedding in to the host image. Unlike traditional watermarking schemes, the second watermarks are not embedded directly into the host image. The proposed technique proves that it is resilient to several attacks.
\end{abstract}

\section{Keyword}

Digital watermarking, visual cryptography, DCT

\section{INTRODUCTION}

Nowadays, the multimedia applications are exponentially growing due to internet revolution. In the cyberspace, the availability of several different methods for copying multimedia data boost the violation of intellectual property rights of digital data, i.e. text, image, audio, and video. Therefore, the prevention of authentic ownership of multimedia data has become an important issue in recent years.

Recently, researchers have proposed various methods to secure the intellectual property rights for digital data. Digital watermarking, a kind of such techniques, is a method that hides a digital binary image or data, called digital watermark, in a host image for the purpose of copyright protection, integrity checking, and captioning. When the authenticate owner of the image needs to be identified, the hidden watermark can be extracted for the ownership verification.

There are two types of watermarking technique are used, visible and invisible. In this paper we used invisible digital watermarking. Invisible (or blind) watermarks, when added to the image can't be perceived as such, and have wider applications than visible watermarks [1].

Watermarks can be embedded in almost every domain (Spatial, DCT, Wavelet, Fourier etc.) using different schemes. While most schemes embed only a single watermark, some extend the single watermark algorithms for multiple watermarks. There are different ways to extract the watermark from the image. Those requiring both the original image and the secret key for the watermark extraction are called private watermark schemes. Those requiring the secret keys but not the original image are called public or blind watermark schemes [2]. Those requiring the secret keys and the watermark are called semiprivate or semi-blind watermark schemes [3]. In general an effective watermarking scheme should satisfy properties such as invisibility, robustness, security, capacity and low computational complexity [4].

This paper proposes multiple watermarking techniques. We used two watermarks, one watermark is directly embedded into a host image using DCT domain and the other watermark is divided into two different shares. First share is kept secret and second share is embedded into host image which is already embedded with first watermark.

The proposed scheme embeds these two watermarks without modifying the original host image. Thus, at no point of time, the watermark information is passed in the transmission channel, thereby providing maximum security.

The remaining part of the paper is organized as follows.

Section 2 briefly reviews the VC scheme. Section 3 describes the basic principle of DCT domain. Section 4 describes the traditional method for watermarking. Section 5 describes proposed Watermark Embedding scheme using VC and DCT. Section 6 describes the watermark embedding and extracting algorithms.

Experimental results are illustrated in Section 7. Section 8 concludes the paper.

\section{VISUAL CRYPTOGRAPHY SCHEME}

Visual Cryptography Scheme (VCS) was first formally introduced by Naor and Shamir [5], which encodes a secret image into $\mathrm{n}$ shares (printed on transparencies) for $\mathrm{n}$ participants.

A visual cryptography scheme for a set $\mathrm{P}$ of $\mathrm{n}$ participants is a method to encode a secret image SI into $\mathrm{n}$ shadow images called shares, where each participant in $\mathrm{P}$ receives one share. Certain quailed subsets of participants can "visually" recover the secret image, but other, forbidden, sets of participants have no information (in an information-theoretic sense) on S I . A "visual" recovery for a set $\mathrm{X}$ consists of Xeroxing the shares given to the participants in $\mathrm{X}$ onto transparencies, and then stacking them. The participants in a qualied set $X$ will be able to see the secret image without any knowledge of cryptography and without performing any cryptographic computation.

\section{PRINCIPLE OF DISCRETE COSINE TRANSFORM DOMAIN}

The DCT based on Mid Band Exchange Coefficient (MBEC) algorithm. DCT-based methods divide image into $8 \times 8$ sized blocks and then transform's image of size $\mathrm{N} \times \mathrm{N}$ into the DCT coefficients matrix with the same size [3]. MBEC use the one bit of binary watermark image. The MBEC watermarking algorithm encodes one-bit of the binary watermark image into one $8 \times 8$

DCT sub-block of the original image. If the difference of two mid-band coefficients is positive in case of the encoded value is " 1 " means the first coefficient is small then second coefficient then we encoded value is " 1 ". Otherwise, these two mid-coefficients are exchanged. 
Classical middle-band based algorithm is quite robust against JPEG compression and common image manipulation operations. The basic idea of the classical MBEC scheme was discussed in [6]

The middle-band frequencies (FM) of an $8 * 8$ DCT block can be shown below in figure 1 . In $8 \times 8$ DCT block the middleband frequencies region is denoted by FM. The lowest frequency component of the block is denoted by FL, while FH is used to denote the higher frequency components. FM is chosen as the embedding region as to provide additional resistance to lossy compression techniques, while avoiding significant modification of the cover image. Next, from the FM region, two locations $\mathrm{Pi}$

(u1, v1) and Pi (u2, v2) are chosen in the ith DCT block for comparison.

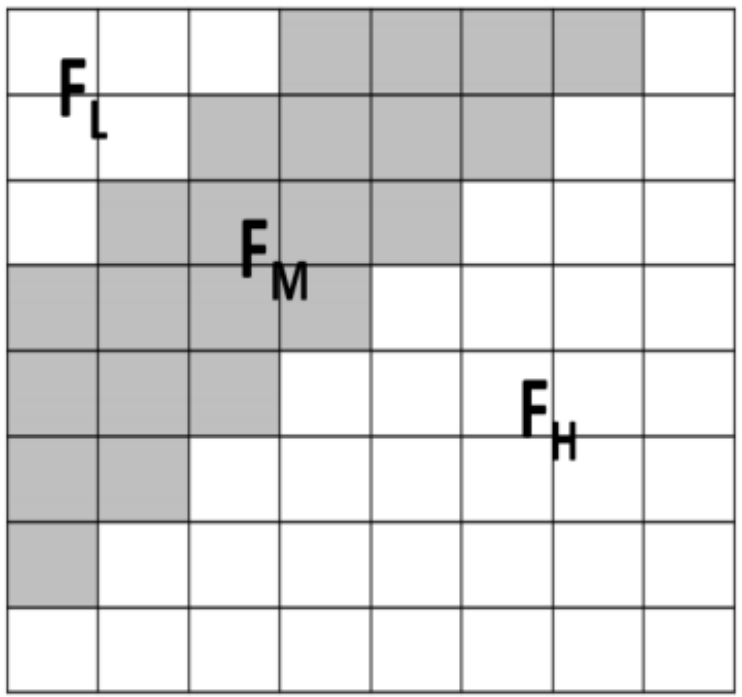

Fig 1: Definition of DCT Region

The equations for two-dimension DCT and Inverse DCT (IDCT) are:

$$
\begin{aligned}
& C(u, v)=a(u) \mathrm{a}(\mathrm{v}) \sum_{x=0}^{N-1} \sum_{y=0}^{N-1} f(x, y) \cos \left(\frac{(2 x+1) u \pi}{2 N}\right) \cos \left(\frac{(2 y+1) v \pi}{2 N}\right) \\
& \mathrm{f}(\mathrm{x}, \mathrm{y})=\sum_{x=0}^{N-1} \sum_{y=0}^{N-1} a(u) \mathrm{a}(\mathrm{v}) \mathrm{c}(\mathrm{u}, \mathrm{v}) \cos \left(\frac{(2 x+1) u \pi}{2 N}\right) \cos \left(\frac{(2 y+1) v \pi}{2 N}\right)
\end{aligned}
$$

Here $\mathrm{C}$ is the DC transformed block, $\mathrm{f}$ is the inverse DCT block and $\mathrm{u}=0,1,2, \ldots, \mathrm{K}-1, \mathrm{v}=0,1,2, \ldots \mathrm{L}-1 ; \mathrm{K}$ and $\mathrm{L}$ are length and breadth of the image. $\mathrm{a}(\mathrm{u})$ and $\mathrm{a}(\mathrm{v})$ are defined in the following equation.

$$
a(u)=\left\{\begin{array}{l}
\frac{1}{\sqrt{K}}, u=0 \\
\sqrt{\frac{2}{K}}, 1 \leq u \leq K-1
\end{array}\right.
$$

$$
a(v)=\left\{\begin{array}{l}
\frac{1}{\sqrt{L}}, v=0 \\
\sqrt{\frac{2}{L}}, 1 \leq v \leq L-1
\end{array}\right.
$$

\section{TRADITIONAL MULTIPLE} WATERMARK EMBEDDING (MWE)

Multiple Watermark Embedding (MWE) extends SWE to embed multiple watermarks in the same image. Multiple watermarks are embedded in the cover image independently, as in SWE. Different secret keys are used with different watermarks to result different Master Shares. These Master Shares are then distributed to the corresponding owners. Note that the watermark is not embedded directly into the digital image. Instead, the cover images information is used to construct the Master Shares. When piracy happens, the detection of multiple watermarks is done independently, using the corresponding author's shares and their secret keys.

\section{PROPOSED METHOD}

Our proposed watermarking scheme complete in two modules. First is the first watermark embedding and second we embedded the first share of second watermark and then we perform watermark extraction process. During the second watermark embedding modules, watermark is split into two shares by means of visual cryptography. Then, one of the two shares is embedded into the DCT domain of the cover image, and the other is distributed to the owner. To prove the ownership, the owner extract his/her first watermark and also extract the other share from the image and then combine these two shares to reveal the watermark. Based on the security condition of visual cryptography, we can make sure that the two shares cannot leak any information about the watermark.

Instead, the proposed method constructs two Master Shares of second watermark for the actual owner according to the original image and the corresponding watermarks. Then, one of the shares is embedded into the host image, and the other is held by the actual owner. When proving the ownership, the actual owner has to recover these two watermarks. First extract watermark is proved the actual owner is authenticate and the second extract watermark that is share one, proved integrity by overlapping these two share one is extract watermark share and another is master share which have actual owner. Unlike other researchers' method, our scheme needs not a trust third party to avoid the multiple calming problems.

\section{WATERMARK EMBEDDING PROCESS}

In Embedding process insert or embed the watermark information within the original image by modifying all or selected pixel values (spatial domain); or coefficients (frequency domain), in such a way that the watermark is undetectable to human eye and is achieved by minimizing the embedding distortion to the host image.

The system block diagram for the embedding process is shown in Figure. 


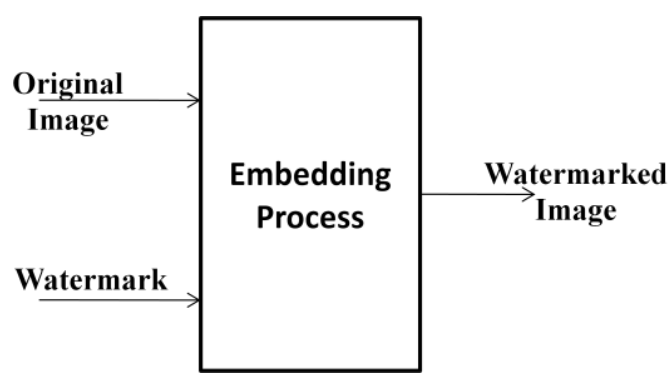

Fig 2: Watermark Embedding process

\subsection{Watermark Embedding Algorithm}

The algorithm which is used to embed a watermark on an image is given below. We take input as Original Image and Watermark data and produce output as Watermarked Image.

Step1: Start

Step2: Read input gray scale Image in $256 \times 256$ standard and Watermark Data.

Step3: Check that the watermark size isn't too large for cover image

if (length(watermark) > length of cover image)

then

error('Message too large to fit in Cover Object') and exit.

Step4: If the Watermark is small then it is padded with ones $\left(1^{\text {ec }} \mathrm{s}\right)$ so the small watermark image scale up to the max message length for original image.

Step5: Perform DCT on Each $8 * 8$ block of image and Embed the watermarking information into the $(5,2)$ and $(4,3)$ pixel of the $8 \times 8$ DCT coefficient block by classical coefficient exchange scheme. DCT of each block is calculated.

Step6: DCT co-efficient at the position say $(5,2)$ and $(4,3)$ are compared for every block. The DCT block will encode a " 0 " if pixel at position $(5,2)$ is greater than or equal to the pixel at the position $(4,3)$ otherwise it will encode a " 1 ". The coefficients are then swapped if the relative size of each coefficient does not agree with the bit that is to be encoded.

Step7: Stop.

\subsection{Watermark Extracting Algorithm}

For check the original image is watermarked or not, after embedding the watermark into original image we apply the watermark extracting algorithm. The algorithm which is used to extract a watermark is given below.

Step1: Start

Step2: the watermarked image transformed into the DCT domain.

Step3: Subdivide the Watermarked image into $8 \mathrm{x} 8$ subimages using DCT domain.

Step4: DCT co-efficient at the position say $(5,2)$ and $(4,3)$ are compared for every block. If pixel at position $(5,2)$ is greater than or equal to the pixel at the position $(4,3)$ then Watermark bit hidden would be black or DCT block will encode a "0" else white or DCT block will encode a "1".
Step5: Stop

\subsection{Share Generating Algorithm Step1: Start}

Step2: Read second watermark as a input.

Step3: Convert watermark in to size of [64x64]

Step4: Initializing the two shares with all values zeros. The width of each share is twice than the width of watermark data.

Step5: Finding all the white pixels indexes in the watermark image where pixel value is equal to 1 .

Step6: For every white pixel we store the required values in each of the share and generate two different shares:

fori $=1:$ len

$\mathrm{a}=\mathrm{x}(\mathrm{i}) ; \mathrm{b}=\mathrm{y}(\mathrm{i})$;

pixel=generateShare(s1 white,s 2 white)

\%dividing the share matrix for white pixels into two shares s1 ((a), $(2 * b-1):(2 * b))=\operatorname{pixel}(1,1: 2)$; $\mathrm{s} 2((\mathrm{a}),(2 * \mathrm{~b}-1):(2 * \mathrm{~b}))=\operatorname{pixel}(2,1: 2)$;

end

Step7: finding all the black pixels indexes in the watermark image

fori $=1:$ len

$\mathrm{a}=\mathrm{x}(\mathrm{i}) ; \mathrm{b}=\mathrm{y}(\mathrm{i})$;

pixel=generateShare(s1black,s2black); \%Getting random permuation of the black set

\%dividing the share matrix for black pixels into two shares s1 ((a), $(2 * b-1):(2 * b))=\operatorname{pixel}(1,1: 2)$

$\mathrm{s} 2((\mathrm{a}),(2 * \mathrm{~b}-1):(2 * b))=\operatorname{pixel}(2,1: 2)$;

end

Step8: Stop

\section{SIMULATION RESULTS AND THEIR ANALYSIS}

Two metrics for quality of watermarked images have been used which are Peak Signal to Noise Ratio (PSNR) and Similarity Factor(SM).

PSNR is defined as:

$$
P S N R=10 \log _{10}\left(\frac{(255)^{2}}{M S E}\right) d b
$$

Where MSE is the mean square error of two images of $\mathrm{N} x \mathrm{~N}$ pixels is defined as

$$
M S E=\frac{1}{N^{2}} \sum_{i=1}^{N} \sum_{j=1}^{N}\left(\mathrm{p}_{i j}-p_{i j}^{\prime}\right)^{2}
$$

Where Pij is the original pixel value and p'ij is the reconstructed pixel value.

The similarity factor has value $[0,1]$ calculated using following equation. If $\mathrm{SM}=1$ then the embedded watermark 
and the extracted watermark are same. Generally value of $\mathrm{SM}>.75$ is accepted as reasonable watermark extraction

$$
S M=\frac{\sum_{i=1}^{M} \sum_{j=1}^{N} W_{M}(i, j) W_{M}^{*}(i, j)}{\sqrt{\sum_{i=1}^{M} \sum_{j=1}^{N} W_{M}(i, j)^{2} \times \sum_{i=1}^{M} \sum_{j=1}^{N} W_{M}^{*}(i, j)^{2}}}
$$

Where WM is Original Watermarkand $\mathrm{WM}^{*}$ is detected watermark

For simulation of proposed watermarking scheme, use the different $512 \times 512$ true color image as the original image and two $64 \times 64$ binary images as watermark image shown in figure 3 .

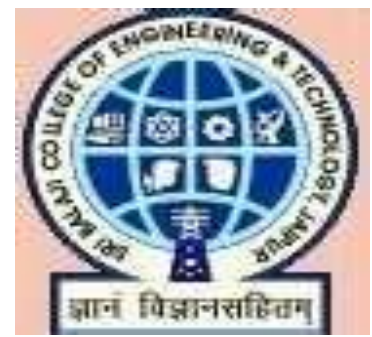

Fig 3: Original first watermark

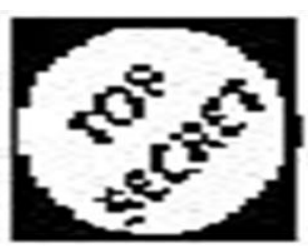

(a)
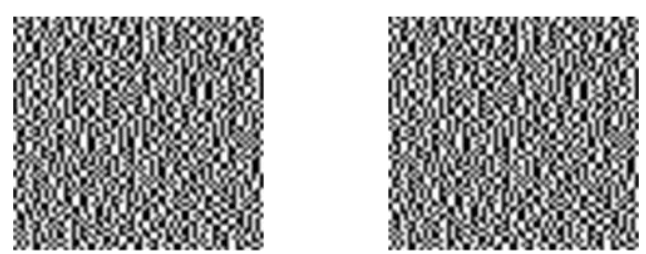

(b)

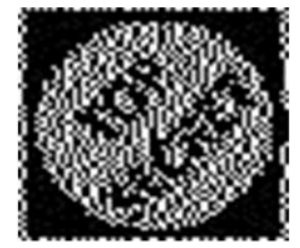

(c)

Fig 4: Second Watermark (a) original two shares (b) overlap these two shares(c)

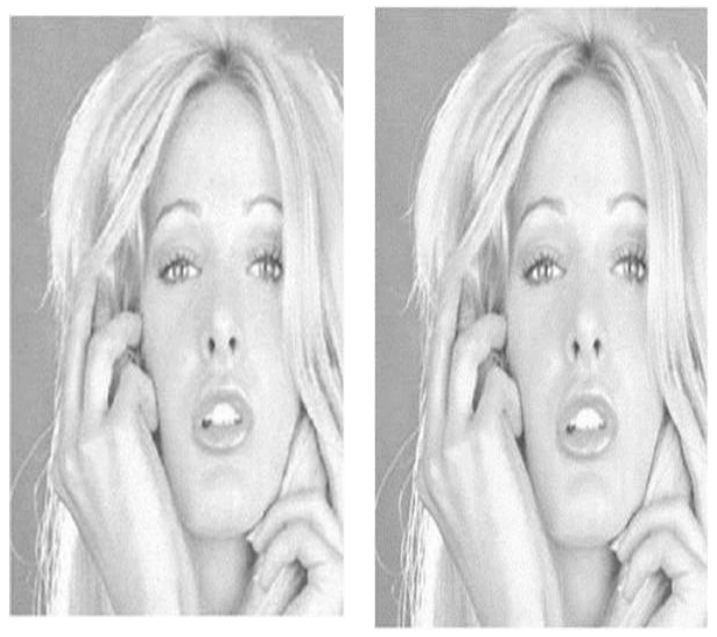

Fig 5: cover 'Girl'image and watermarked 'Girl' image

Table 1. PSNR and SM value for 'Girl' Image

\begin{tabular}{|c|c|c|c|}
\hline Image & PSNR (in db) & $\begin{array}{c}\text { SM of First } \\
\text { watermark }\end{array}$ & $\begin{array}{c}\text { SM of } \\
\text { Second } \\
\text { Watermark }\end{array}$ \\
\hline Girl & 41.87 & 1 & 1 \\
\hline
\end{tabular}

We also compare different PSNR and SM value for Girl image. The following table shows the value of PSNR and SM in different JPEG attack.

Table 2. Effect of JPEG attacks

\begin{tabular}{|l|l|l|l|}
\hline $\begin{array}{l}\text { JPEG } \\
\text { Attacks }\end{array}$ & $\begin{array}{l}\text { PSNR (in } \\
\mathrm{db})\end{array}$ & $\begin{array}{l}\text { SM of First } \\
\text { watermark }\end{array}$ & $\begin{array}{l}\text { SM } \\
\text { Second } \\
\text { Watermark }\end{array}$ \\
\hline 10 & 31.55 & 0.86 & 0.67 \\
\hline 20 & 33.80 & 0.79 & 0.65 \\
\hline 30 & 34.81 & 0.72 & 0.62 \\
\hline 40 & 35.43 & 0.73 & 0.65 \\
\hline 50 & 35.95 & 0.75 & 0.68 \\
\hline 60 & 36.50 & 0.78 & 0.73 \\
\hline 70 & 37.26 & 0.84 & 0.80 \\
\hline 80 & 38.17 & 0.88 & 0.85 \\
\hline 90 & 39.53 & 0.94 & 0.91 \\
\hline 100 & 41.71 & 0.96 & 0.96 \\
\hline
\end{tabular}




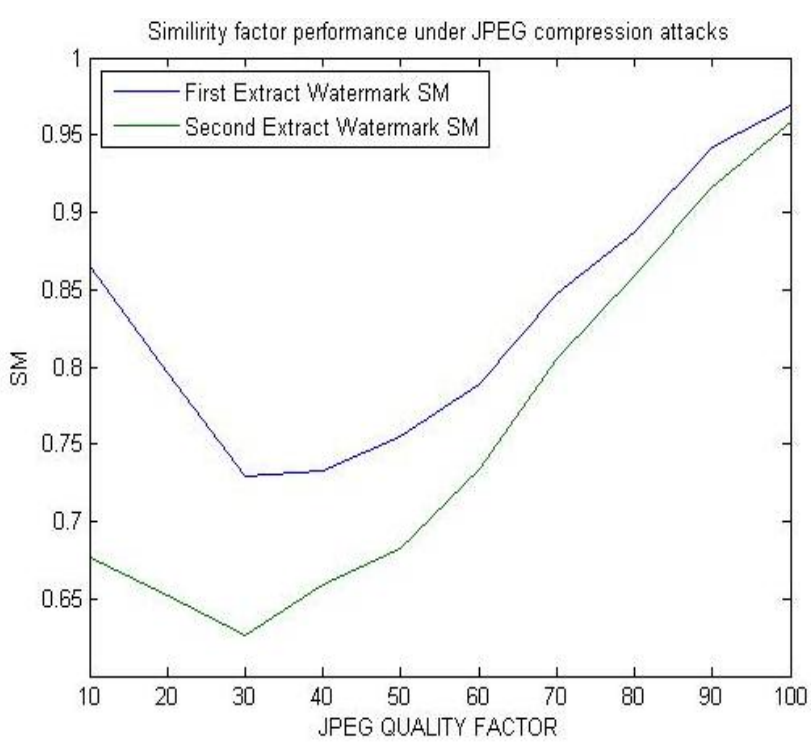

Fig 6: Similarity factor performance under JPEG compression attacks

\section{CONCLUSION}

This paper proposes an image watermarking scheme based on visual cryptography in discrete cosine transform (DCT). Experiments are showed to demonstrate that the proposed scheme is robust against JPEG compression. The proposed scheme can identify the ownership without the original host image and it does not alter the host image to hide the invisible watermark. It is not possible to recover the invisible identification share without the secret key in the proposed scheme. The security requirement of the proposed algorithm is achieved with the visual cryptography.

\section{REFERENCES}

[1] Braudaway G. W., K. A. Magerlein, and F. Mintzer.1996, Protecting Publicly-available Images with a Visible Image Watermark, in the Proceedings of SPIE, 2659, 126-133.

[2] Cox, I. J.,M. L. Miller, and J. A. Bloom. 2002, Digital Watermarking, New York: Morgan Kaufmann Publishers Inc., San Fransisco, CA.

[3] Kutter M. and F. A. P. Petitcolas. 1999, A fair benchmark for image watermarking systems, In Proceedings of Security and Watermarking of Multimedia Contents, 226-239.

[4] Cox, I. J., Kilian, J., Leighton, T., and Shamoon, T..1997, Secure Spread Spectrum Watermarking for Multimedia, In IEEE Transactions on Image Processing, 6(12), pp. 1673-1687.

[5] M. Naor and A. Shamir.Visual cryptography. In EUROCRYPT '94, Springer-VerlagBerlin, volume LNCS 950, pages $1\{12,1995$.

[6] S. F. Tu and C. S. Hsu, "A BTC-based watermarking scheme for digital images," Information \& Security: An International Journal, vol. 15, no. 2, 2004, pp. 214-226. 